\title{
Electron bremsstrahlung recovery in ATLAS tracking using Dynamic Noise Adjustment
}

\author{
Evelina Bouhova-Thacker, Vakhtang Kartvelishvili* \\ Lancaster University, UK (on behalf of ATLAS collaboration) \\ E-mail: vakhtang.kartvelishvili@cern.ch
}

\begin{abstract}
A number of approaches are being used in ATLAS reconstruction software, aiming to improve electron momentum measurement by taking into account the effects due to bremsstrahlung at various stages. One of them, designed and implemented in ATLAS, is covered in this talk. The method applies Dynamic Noise Adjustment (DNA) during the track fitting process based on the Kalman filter, and is both fast and efficient.
\end{abstract}

XI International Workshop on Advanced Computing and Analysis Techniques in Physics Research April 23-27 2007

Amsterdam, the Netherlands

\footnotetext{
* Speaker.
} 


\section{Introduction}

Various physics channels to be used for physics studies by the ATLAS collaboration at the LHC - Higgs and supersymmetry searches, B-hadron and quarkonium physics and many others - require effi cient detection and precise measurement of electrons and positrons produced in proton-proton collisions. High precision silicon detectors, transition radiation detectors and electromagnetic calorimeters are used for this purpose in the ATLAS detector (see fig. 1 and ref. [1] for details).

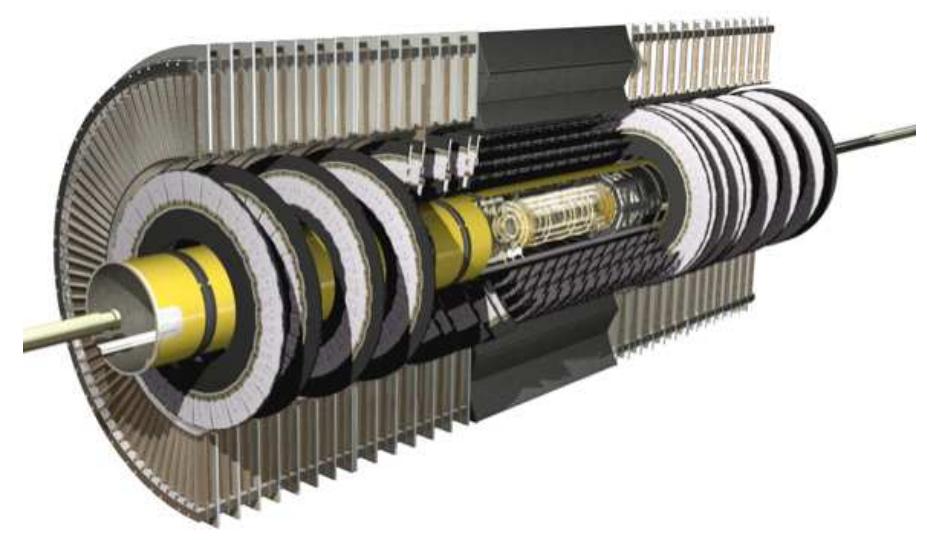

Figure 1: Schematic view of the ATLAS Inner Detector.

However, the amount of material being traversed by the electrons propagating through the ATLAS inner detector (ID), shown in fi g. 2, is not negligible. In particular, particles emerging

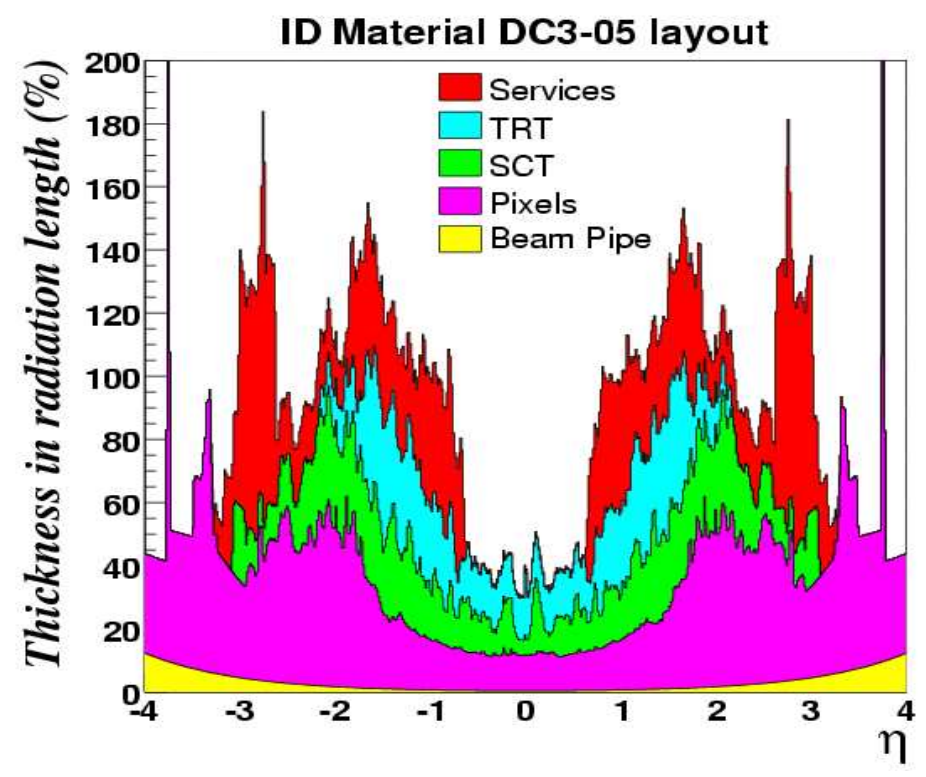

Figure 2: Material budget of ATLAS ID. The amount of material traversed by the electrons varies with pseudorapidity $\eta$ from about 0.5 to about 1.5 radiation lengths. 
from the interaction point in the pseudorapidity range $|\eta|<0.8$ ("barrel region") will go through the beam pipe, 3 layers of Pixel detectors, 4 layers of Semi-Conductor Tracker detectors (SCT), and 73 layers of Transition Radiation Detector (TRT) before reaching the calorimetry. Even in the barrel region, every layer of the high precision silicon detectors (Pixel and SCT) contributes 2 to 6 percent of $X_{0}$. This results in a high probability of signifi cant bremsstrahlung radiation by electrons and positrons, influencing the quality of their track reconstruction and measurement.

\section{Bremsstrahlung}

The "brem-ray" image of the ATLAS Inner detector (fi g. 3), which shows $R-Z$ coordinates

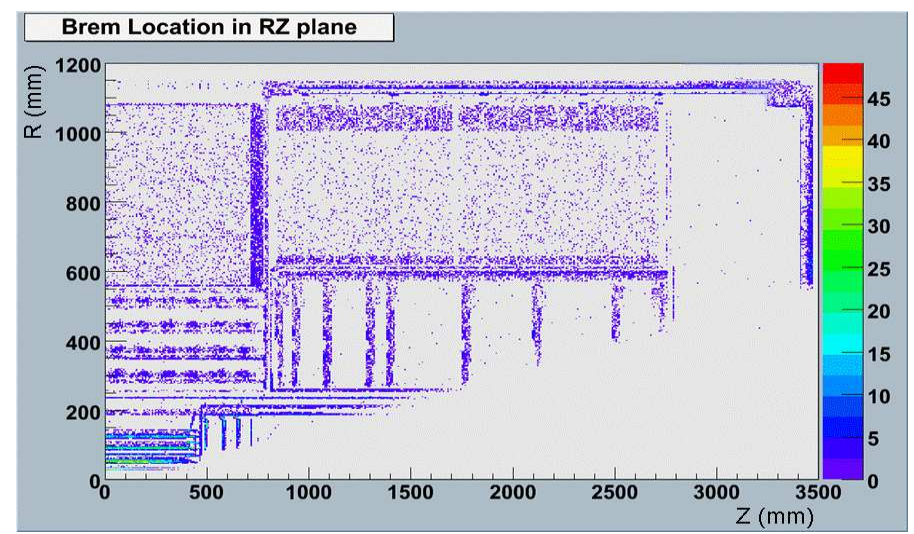

Figure 3: The $R-Z$ coordinates of simulated bremsstrahlung points, showing that the denser parts of the inner detector account for more frequent brems, with more energy radiated.

of simulated bremsstrahlung points, highlights the fact that denser parts of the detector account for more frequent brems, with more energy radiated. Early brems - in pixel and lower silicon layers - make electron momentum measurement ever more diffi cult.

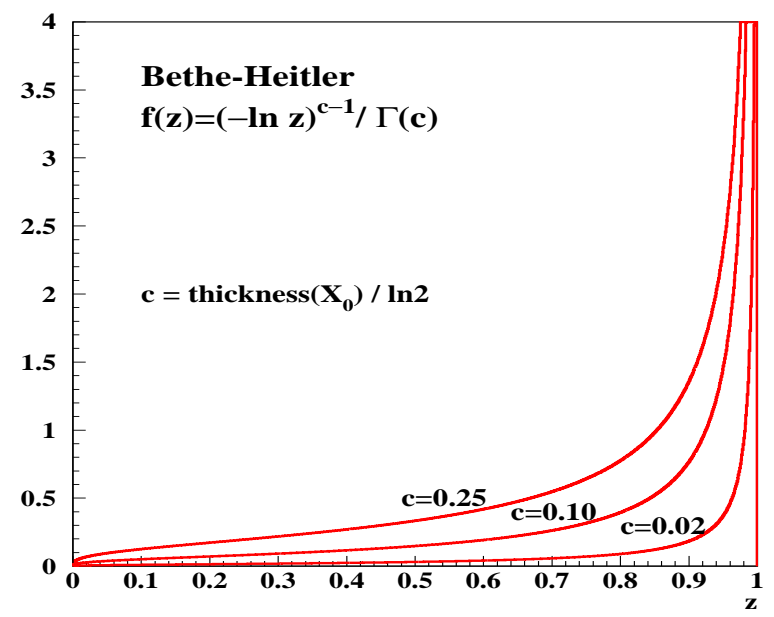

Figure 4: Bethe-Heitler distribution of the fraction $z$ of energy retained by the radiating electron. 
According to the description of the bremsstrahlung process [2], the electron retains its direction of propagation and a fraction $z$ of its energy, with the probability density given by the Bethe-Heitler distribution (fi g.4). This probability density $f(z)$ depends on the amount of traversed material and is highly asymmetric, with a singularity at $z \rightarrow 1$ and a long tail extending to very small $z$.

Fig. 5 shows a radiating track in the magnetic fi eld. If an electron suffers an act of bremsstrahlung at point $C$, its track will have a smaller radius of curvature after that point, hitting layer S5 at point $\mathrm{F}$ instead of point $\mathrm{G}$ (while the photon follows the tangent $\mathrm{C}-\mathrm{E}-\mathrm{H}$ ). A tracking algorithm which

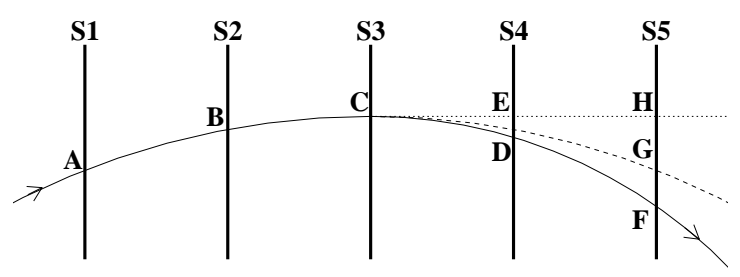

Figure 5: A track changing its curvature at point $C$ due to bremsstrahlung. This track has lost $35 \%$ of its momentum at that point.

does not take the possibility of such changes into account will, in such cases, return a track with an underestimated momentum value and unacceptable fi t quality.

\section{Kalman Filtering}

The default track reconstruction method in ATLAS [3] uses a Kalman filter (KF) [4, 5]. This is an iterative procedure used to determine the vector of track parameters, $y$, and its covariance matrix, $C$, taking into account the measurement $m$ (and its respective covariance matrix $V$ ) at each detector layer $k$. In a slightly simplifi ed notation, the procedure includes three steps:

1. Extrapolate $y$ and $C$ from layer $k-1$ to layer $k$ :

$$
y_{k-1} \rightarrow y_{k}^{-}, \quad C_{k-1}+Q_{k-1} \rightarrow C_{k}^{-}
$$

Here $Q_{k}$ is the covariance matrix of the system noise at layer $k$, which can take into account things like multiple scattering and bremsstrahlung.

2. Calculate Kalman gain $K$ at layer $k$ :

$$
K_{k}=\frac{C_{k}^{-}}{C_{k}^{-}+V_{k}}
$$

3. Do measurement update:

$$
y_{k}=y_{k}^{-}+K_{k}\left(m_{k}-y_{k}^{-}\right), \quad C_{k}=\left(1-K_{k}\right) C_{k}^{-}
$$


It can be shown (see e.g. [4] and references therein) that this procedure only works properly if both measurement uncertainty $V$ and the process noise $Q$ are well described by gaussian distributions. While this is usually the case for measurement errors and multiple scattering, it's certainly not true for bremsstrahlung, which causes problems during electron track reconstruction. As mentioned above, when applied to a radiating electron track similar to the one shown in fig. 5 , the default KF fi t is likely to result in a bad fi t quality with an underestimated reconstructed momentum.

\section{Gaussian Sum Filter}

In the standard implementation, the Kalman fi lter can only view bremsstrahlung as another source of gaussian-distributed noise, which is clearly a very crude approximation to the BetheHeitler distribution (fi g. 6). So, early attempts to replace the Bethe-Heitler distribution with a

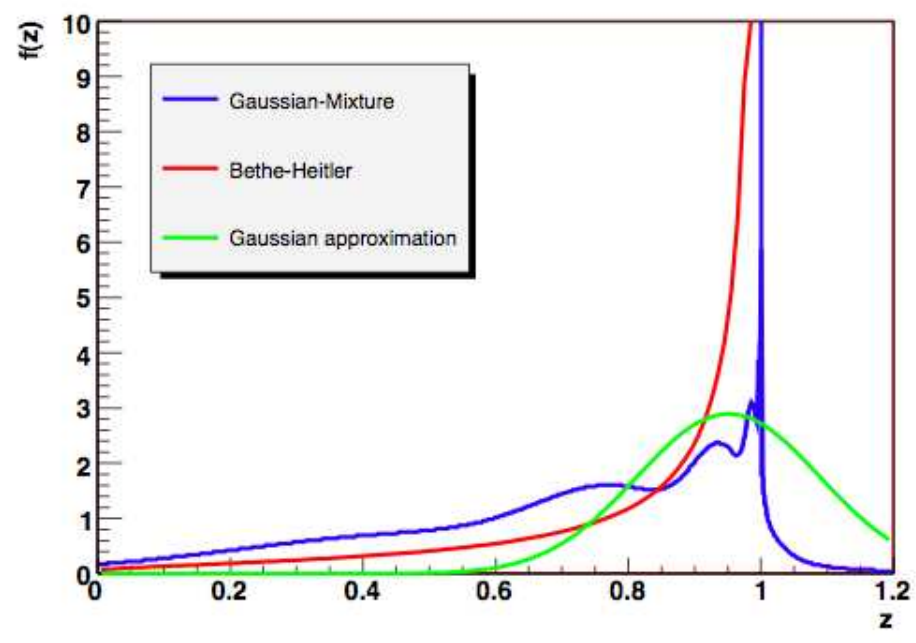

Figure 6: Comparison between the Bethe-Heitler distribution, a single gaussian distribution of equivalent mean and r.m.s., and a sum of several gaussians used by the Gaussian Sum Filter.

single gaussian of equivalent mean and width did not work too well.

The Gaussian Sum Filter (GSF) [4, 6] is a non-linear generalisation of the Kalman fi lter. GSF approximates Bethe-Heitler distribution by a mixture of several gaussians, thus allowing to take into account the asymmetry and the low-energy tail of the distribution. After that, several Kalman fi lters run in parallel. In order to make things manageable and avoid exponential growth of components, component reduction has to be performed at each step. Even so, GSF may take 10 or even 100 times longer than the default KF.

GSF can be quite successful in bremsstrahlung recovery - e.g., the effective track momentum resolution for $10 \mathrm{GeV}$ electrons in ATLAS improves from about $10 \%$ to $8 \%$. However, the hefty computing overhead is a major limiting factor in its use.

\section{Dynamic Noise Adjustment}

Another, quick and effi cient method of dealing with electron tracks has been developed and 
implemented in ATLAS. It is based on the Dynamic Noise Adjustment (DNA) [7] during Kalman fi ltering.

At each silicon layer, a simple single-parameter fit is performed to flag hits which may be associated with bremsstrahlung. This fit tries to estimate the increase in curvature due to possible bremsstrahlung at the current detector layer. If no bremsstrahlung was flagged, the track fitter reverts to the default Kalman fi ltering procedure. Otherwise, the result of the single parameter fi t - the estimated fraction of energy retained by the electron, $z$ - is used to calculate the additional effective "system noise" term, which is then fed to the Kalman fi lter.

The effective "system noise" variance calculation is illustrated by fi g. 7, which shows how the Bethe-Heitler distribution is mapped onto the gaussian distribution of unit width. The deviation $\Delta z$ of the estimated $z$ from the median $z_{0}$ is mapped onto the gaussian to fi nd the corresponding deviation $\Delta x$. The effective noise $\sigma$ is then calculated as $\sigma_{\mathrm{DNA}}(z)=\Delta z / \Delta x$. This procedure is equivalent
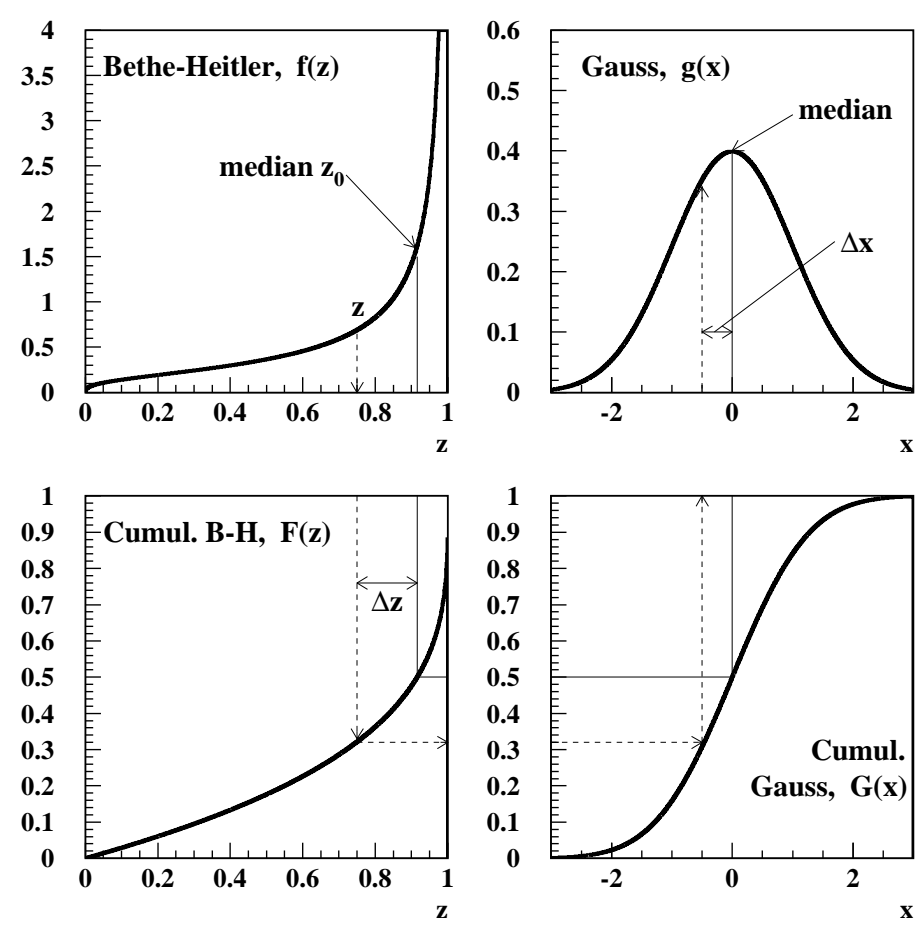

Figure 7: Mapping of the probability distributions used to calculate the variance of the effective noise term.

to representing the random variable $z$, distributed according to the Bethe-Heitler probability density, in the form

$$
z=z_{0}+x \sigma_{\mathrm{DNA}}(z)
$$

where $x$ is a random variable with gaussian probability distribution.

So, in this approach bremsstrahlung is legitimately treated as a source of gaussian noise, but only in those cases when bremsstrahlung-like behaviour of the track has been detected. The proper gaussian probability distribution of this "system noise" is now guaranteed by construction. 
Of course, $\sigma_{\mathrm{DNA}}$ also depends on the thickness of material associated with the corresponding layer, as illustrated by Fig. 8 .

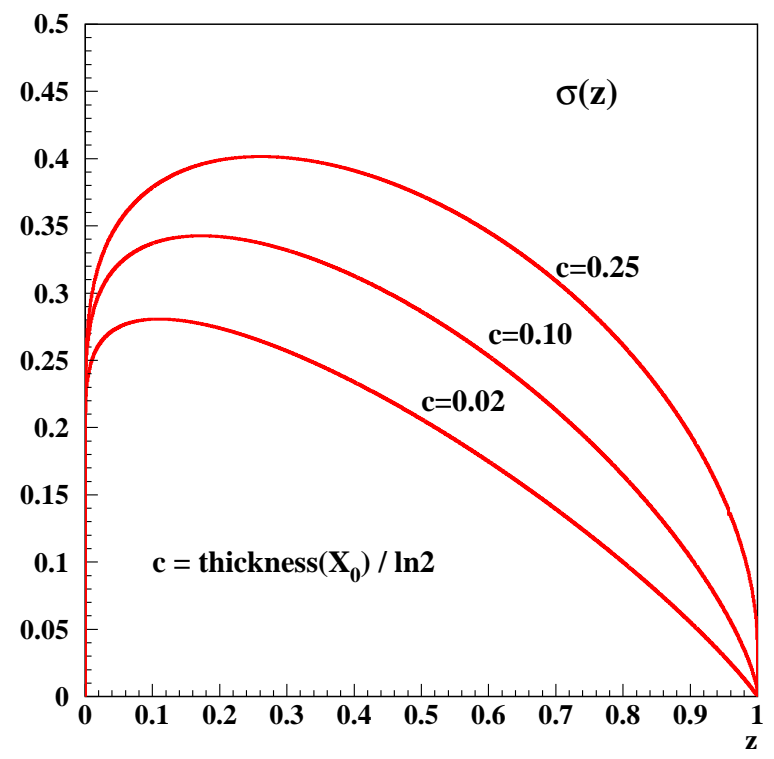

Figure 8: Effective $\sigma_{\mathrm{DNA}}(z)$ for various values of material thickness in the detector layer.

The variance $\sigma_{\mathrm{DNA}}^{2}$ is added to the appropriate term of the Kalman covariance matrix used during the fi t, similarly to the treatment of any other source of system noise. However, this particular noise term is adjusted dynamically according to the estimated $z$ and the thickness of the layer, thus justifying the name of the method.

\section{DNA fit in action}

The comparison between the results of the default Kalman Fit (labelled "DEFAULT", top row) and KF with DNA (labelled "DNA fi t", bottom row), for a simulated sample of $20 \mathrm{GeV}$ single electrons in the barrel region $|\eta|<0.8$, is shown in Fig. 9. The left column shows the ratio of measured to generated momentum, where one can see a marked reduction of the low momentum tail and a signifi cant improvement in the average momentum measurement. The distribution of estimated errors on measered momentum, returned by the fi t (middle column), shows an increase of the errors in cases where radiation has been flagged, which is quite natural and signifi cantly increases the percentage of reliably measured electron tracks. This is illustrated by the improvement in the pull distribution (right column) with the gaussian core now showing a mean of -0.1 and a width of 0.98 (as opposed to -0.6 and 1.09 , respectively, for the default fi t).

When applied to heavier particles, the DNA fi $t$ did result in some deterioration of the fi t quality, and introduced some small biases, which we hope to reduce in the future. Investigation of the differences in the behaviour of the fi tted momentum for electron and pion/muon tracks, with and without the DNA corrections, shows some interesting patterns, which open the possibility of separating "false positives" from true radiating electrons and may result in a useful tool for early identifi cation of electrons. 

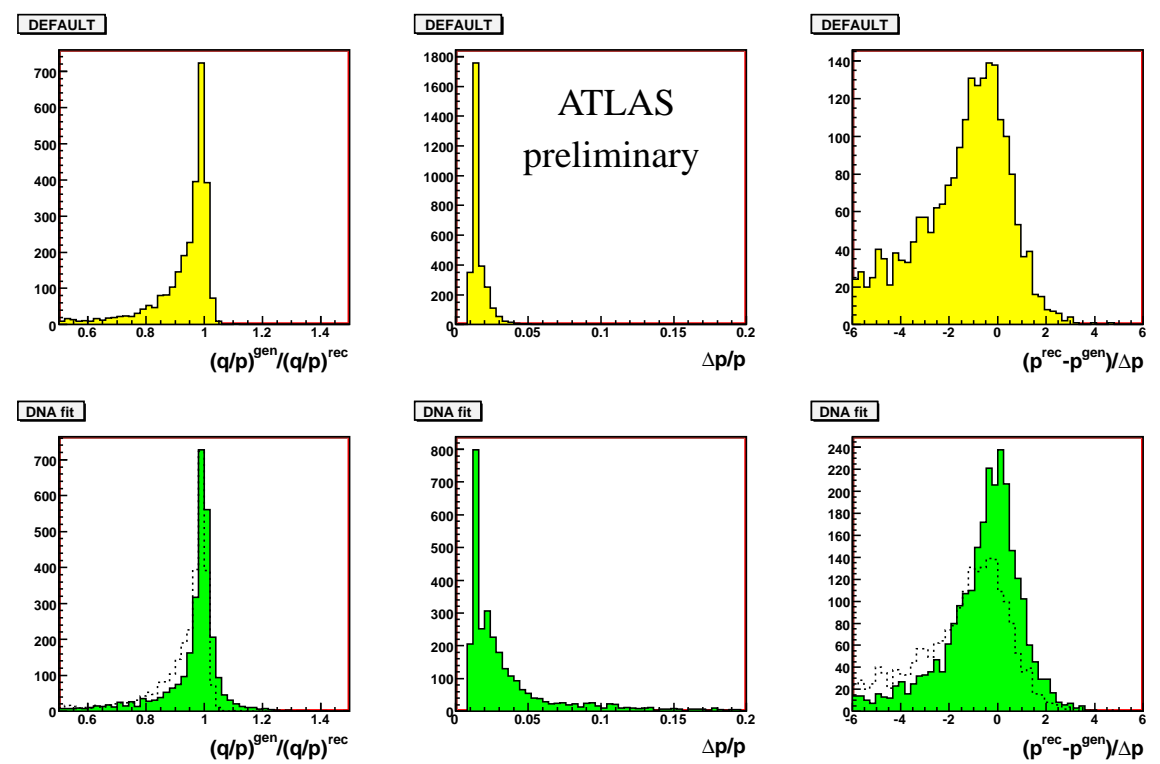

Figure 9: Comparison of measured momentum, estimated error and pull distributions with default fi $t$ (top row) and DNA fi t (bottom row) for single electron simulations at $20 \mathrm{GeV}$ in the barrel region.

Table 1 shows the comparison of the numbers of simulated electron tracks in various selections, for the default and DNA fi ts. Note that improved track measurement in the precision silicon layers results in better matching between the silicon and TRT track segments. This, in turn, reduces the fraction of electron tracks without TRT extensions from $20 \%$ to $8 \%$. This also means better extrapolation to the electromagnetic calorimeter (ECAL) and hence improved track-to-ECAL matching.

Table 1: Fractions of electron tracks falling into various selections, for the default $K F$ and the DNA fi ts.

\begin{tabular}{|l||c|c|}
\hline Selection & Default KF & with DNA \\
\hline Total reconstructed tracks & $99.0 \%$ & $99.4 \%$ \\
Momentum within $\pm 50 \%$ of true & $93.4 \%$ & $94.5 \%$ \\
Momentum within $\pm 20 \%$ of true & $84.2 \%$ & $86.4 \%$ \\
Within gaussian core of $q / p$ distr. & $55.6 \%$ & $65.5 \%$ \\
Within gaussian core of pull distr. & $51.3 \%$ & $71.2 \%$ \\
Tracks with TRT extensions & $80 \%$ & $92 \%$ \\
\hline
\end{tabular}


DNA fi t only works with precision hits from silicon layers, and hence cannot help in recovering electron tracks radiating in TRT. For very high energy electrons its effectiveness is also reduced, as the curvature radii become too large. However, the results of the DNA fi t can be used as a good starting point for yet another method of bremsstrahlung recovery, being currently developed in ATLAS. The latter uses the information from the matched ECAL cluster to improve the track fi t, and thus can address these problem areas.

Remarkably, the computing overhead of the DNA fi t is quite small, below $10 \%$ on top of the default fi t. Better segment matching means fewer iterations of the Kalman fi tter, thus sometimes making the DNA fi t even faster than the default one. This opens the possibility of using the DNA fi t during online processing in the high-level trigger, thus improving the electron trigger effi ciency.

First results on full simulation and reconstruction of $J / \psi \rightarrow e^{+} e^{-}$in $B$ decay events (fi g. 10) show a clear improvement if DNA brem recovery is performed: the $15 \mathrm{MeV}$ shift of the $J / \psi$ mass

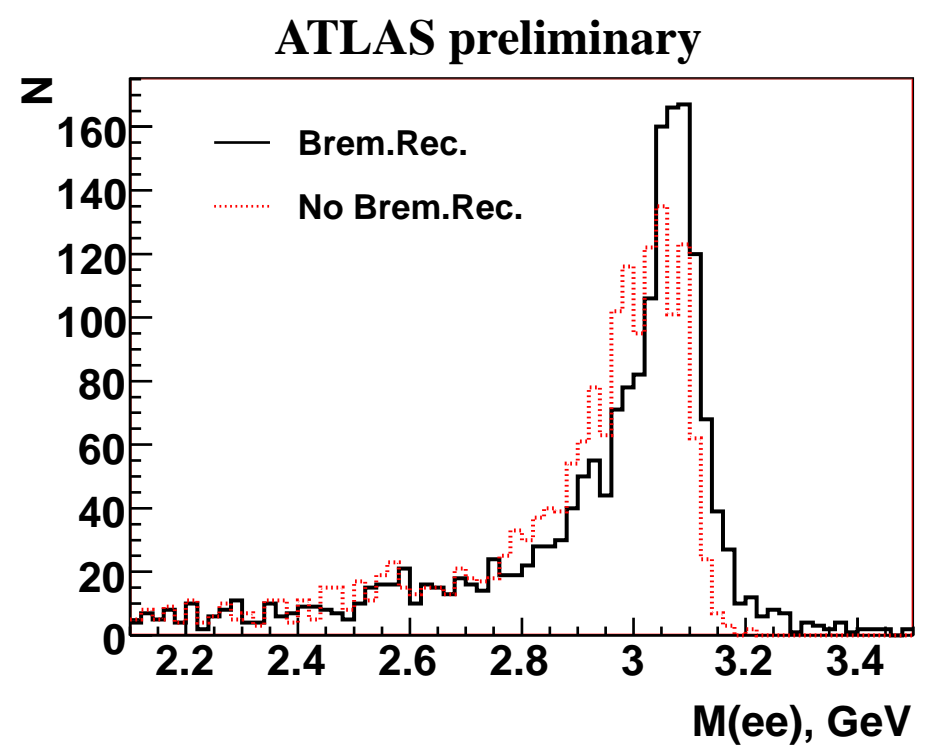

Figure 10: Reconstructed invariant mass of $e^{+} e^{-}$in $B \rightarrow J / \psi\left(\rightarrow e^{+} e^{-}\right)+X$ decay events, with (solid line) and without (dashed line) bremsstrahlung recovery using the DNA fit.

virtually disappears, invariant mass resolution is better by about 20\%, and the $J / \psi$ reconstruction effi ciency is increased by 5\%. It's worth noting that more reliable error estimates returned by the DNA fi t play an important role in improving the fit of the secondary vertex, necessary for this analysis.

\section{Conclusion}

Bremsstrahlung recovery is extremely important in order to achieve the full range of physics goals in ATLAS. Higgs decays $H \rightarrow Z Z \rightarrow e^{+} e^{-} e^{+} e^{-}$and SUSY searches in multi-leptonic fi nal states are good examples of processes to benefi $t$ from it. Hence the number of different approaches to bremsstrahlung recovery that have been developed, tried and implemented in ATLAS. 
This presentation described the track fi tting procedure which uses Dynamic Noise Adjustment during the Kalman fi ltering process, used in ATLAS tracking. DNA fi t works well: signifi cant improvements in electron track reconstruction effi ciency and quality have been achieved. Thanks to its speed, DNA fit can also be used online in high-level trigger to improve electron trigger effi ciency.

DNA fi $\mathrm{t}$ is still in development, with further tests and potential improvements in progress. For example, in some cases "early" electron identifi cation, based on silicon hits, may become possible.

\section{Acknowledgements}

The author is grateful to many ATLAS colleagues who contributed to this presentation one way or another. Special thanks are due to Tom Atkinson, Anne-Catherine Le Bihan, Maria Jose Costa, Markus Elsing, Igor Gavrilenko, Jason Lee, Wolfgang Liebig, Darren Price and Andreas Salzburger.

\section{References}

[1] ATLAS Detector and Physics Performance TDR, CERN/LHCC 99-14, Geneva, May 1999.

[2] H. Bethe, W. Heitler, Proc. Roy. Soc. Lond. A146 (1934) 83.

[3] ATLAS tracking event data model. F. Akesson et al., ATL-SOFT-PUB-2006-004, CERN, Geneva, July 2006.

[4] Branko Ristic, Sanjeev Arulampalam, and Neil Gordon, 'Beyond the Kalman Filter: Particle Filters for Tracking Applications", Artech House, 2004.

[5] R. Frühwirth, Nucl. Instr. Meth. A262 (1987) 444.

[6] R. Frühwirth, Comp. Phys. Comm. 100 (1997) 1.

[7] V. Kartvelishvili, 'Electron bremsstrahlung recovery in ATLAS”, talk given at IPRD06, 1-5 October 2006, Siena, Italy. 